\title{
A formação do técnico em enfermagem: perfil de qualificação
}

\author{
Nursing technician training: qualification profile
}

Mônica Carvalho de Mesquita Werner Wermelinger (https://orcid.org/0000-0001-5861-5479) ${ }^{1}$

Anderson Boanafina (https://orcid.org/0000-0002-3567-7172) ${ }^{2}$

Maria Helena Machado (https://orcid.org/0000-0002-5209-2424) ${ }^{1}$

Monica Vieira (https://orcid.org/0000-0002-5935-2938) ${ }^{3}$

Francisco Rosemiro Guimarães Ximenes Neto (https://orcid.org/0000-0002-7905-9990) ${ }^{4}$

Wagner Ferraz de Lacerda (https://orcid.org/0000-0002-3388-5314) ${ }^{1}$

${ }^{1}$ Escola Nacional de Saúde Pública Sergio Arouca, Fundação Oswaldo Cruz. R. Leopoldo Bulhões 1480, Manguinhos. 21041-210 Rio de Janeiro RJ Brasil. monicaw@ensp.fiocruz.br

${ }^{2}$ Casa de Oswaldo Cruz, Fiocruz. Rio de Janeiro RJ Brasil.

${ }^{3}$ Escola Politécnica de Saúde Joaquim Venâncio, Fiocruz. Rio de Janeiro RJ Brasil.

${ }^{4}$ Universidade Estadual Vale do Acaraú. Sobral CE Brasil.

\begin{abstract}
The identification of the current vocational training profile of nursing aides and technicians becomes a central element in understanding the dynamics of their qualification in several states, aiming to expose both the existence of trends for under- and overqualification and the participation of the public sector in the offer and expansion of nursing courses in the country. The article explores three relevant aspects of vocational training based on the results found in the research "Nursing Profile in Brazil (FIOCRUZ/ COFEN)": the level of schooling/qualification; the geographical distribution and the governmental participation in the consolidation of the current situation. This is an analytical study based on the interpretation of indicators identified by Pearson's Asymmetry Coefficient. The study uses the database generated by the research, as well as data from MEC/Inep and IBGE. The achieved results establish relations between the characteristics of training, distribution of NA\&T in all Brazilian states with the phenomenon of overqualification, besides revealing an apparent separation of the Federal Education Network from the actual demand for nursing technicians in the country.

Key words Nursing profile, Nursing training, Under- and overqualification, Federal Education Network
\end{abstract}

Resumo A identificação do atual perfil de formação profissional de auxiliares e técnicos de enfermagem (A\&TE) se torna elemento central na compreensão da dinâmica da qualificação desses trabalhadores em diferentes Estados, com o objetivo de revelar tanto a existência de tendências para sub e sobrequalificação, quanto a participação do setor público na oferta e expansão dos cursos de enfermagem no país. O artigo explora três aspectos relevantes da formação profissional a partir dos resultados encontrados na pesquisa "Perfil da Enfermagem no Brasil (FIOCRUZ/COFEN)": o nivel de escolaridade/qualificação; a distribuição geográfica e a participação governamental na consolidação do quadro atual. Trata-se de um estudo analítico baseado na interpretação de indicadores definidos pelo Coeficiente de Assimetria de Pearson. O estudo utiliza o banco de dados gerado pela pesquisa, além de dados do MEC/Inep e do IBGE. Os resultados alcançados estabelecem relações entre as características de formação, distribuição de A\&TE em todos os estados brasileiros com o fenômeno da superqualificação, além de evidenciar um aparente descolamento da Rede Federal de Educação com a real demanda por técnicos de enfermagem no país.

Palavras-chave Perfil da enfermagem, Formação em enfermagem, Sub e sobrequalificação, Rede Federal de educação 


\section{Introdução}

Se for verdade que a Reforma Universitária brasileira perpetrada nos anos de 1960 e 1970 - tida como "desdobramento do milagre brasileiro na esfera educacional", mas que "meramente contribuiu para alargar um pouco mais o restrito número de privilegiados que atingem o ápice da pirâmide"1 - nada tinha de democrática nem de democratizante, além de não alterar a qualidade do ensino superior, 'seja quanto à intensidade, à racionalidade e ao rendimento'; também é verdade afirmar que, hoje, essa arena de conflitos e interesses é palco de um processo construído desde 2003 e que a realidade educacional, na última década, foi instituindo um sem-número de projetos, programas e políticas governamentais que, ora buscam a expansão universitária, ora a inclusão, acesso e permanência de camadas populacionais específicas no ensino superior (Prouni, Fies, Sisu, além de programas especiais).

Esse contexto repercute, em certa medida, na corrida pelo acesso aos cursos com a finalidade de obtenção de 'diplomas' que, na conjuntura das incertezas do mercado, foram se tornando requisito de acesso e permanência no emprego ${ }^{1}$. Contudo, enquanto o acesso à educação se torna, a cada dia, mais decisório para a inserção dos jovens e adultos no mundo produtivo, para a população que vive distante dos grandes centros urbanos esse acesso permanece difícil, pela falta de instituições nessas regiões. Quando o tema está relacionado com a saúde e a vida, a questão da qualificação ganha novos e relevantes contornos.

No cenário nacional, a participação dos enfermeiros na equipe de enfermagem corresponde a $23 \%$ e auxiliares e técnicos, $77 \%{ }^{2}$. Esse é um salto de qualificação se pensarmos que, a menos de $40 \mathrm{anos}^{3}$, os atendentes representavam 63,8\% da equipe e os enfermeiros apenas $8,5 \%$. Esta ampliação do acesso aos cursos de enfermagem ocorreu de forma concomitante a outros dois fenômenos associados: a redução da participação de atendentes na equipe; e o aumento da escolaridade entre Auxiliares e Técnicos de enfermagem (A\&TE). Em 1983³ , 9,6\% dos A\&TE tinham nível superior completo ou incompleto; 30 anos depois, segundo os dados da Pesquisa Perfil da Enfermagem no Brasil (PPEB), esse percentual passou para $34,3 \%{ }^{2}$.

Considerando a especificidade da organização da enfermagem em categorias profissionais distintas; a atual exigência da formação técnica de nível médio como patamar mínimo para inscrição no Conselho profissional; e a expressiva participação de A\&TE na equipe, a questão da formação desses profissionais reveste-se de complexidade, impondo a escolha de aspectos centrais que permitam compreender o seu quadro atual.

A análise dos resultados da $\mathrm{PPEB}^{2}$ que caracterizam o quadro atual dessa formação nos diversos estados foi estruturada a partir de três dimensões associadas: a distribuição geográfica, $o$ nível de escolaridade/qualificação dos A\&TE e a participação governamental na consolidação do quadro atual. Assim, buscaremos respostas para questões como: Há sobrequalificação ou subqualificação de técnicos ou, ainda, há uma convivência dos dois fenômenos? Quais os estados com maiores/menores índices de qualificação desses técnicos? Qual foi a participação do Poder Público federal na formação de técnicos nos estados?

Os resultados encontrados precisam ser contextualizados na história recente de expansão do acesso ao ensino vivenciada em nosso país. Nesse sentido cabe compreender que, para além de um instrumento político e ideológico de resposta às exigências dos mercados, a educação e, em especial, a profissional, devem ser tomadas como baluarte do desenvolvimento social com justiça e bem-estar.

\section{A Questão da Qualificação Profissional}

O rápido avanço dos processos de urbanização e desenvolvimento tecnológico vem promovendo a ruptura de fronteiras físicas e culturais. Contudo, de forma antagônica, os mesmos processos estão ampliando a distância entre os incluídos e os excluídos socialmente. Neste contexto, os requisitos de escolarização estão atrelados a um projeto de sociedade que sustente e perpetue a divisão entre os que possuem os meios de produção e os que não possuem ${ }^{4}$; ampliando, ao longo dos anos, a "desigualdade econômica entre os 'organizadores' - cada vez mais exploradores - e os 'executores' - cada vez mais explorados"s. $\mathrm{Na}$ sociedade capitalista, a escola é uma "engrenagem dentro do sistema" $\mathrm{e}$ a educação reflete os anseios da classe dominante que mantém, através da propriedade do saber, a hegemonia política e a liderança intelectual como forma de domínio e de controle social, influenciando, principalmente, $\mathrm{o}$ arcabouço legal que sustenta as diretrizes para a formação do trabalhador.

Seguindo esta linha de pensamento é possível constatar que o novo tipo de produção, racionalizada, requer do trabalhador adequação "aos novos métodos de trabalho caracterizados pela 
automação, ou seja, pela ausência de mobilização de energias intelectuais e criativas no desempenho do trabalho"'. Portanto, uma educação que desenvolva a consciência crítico-reflexiva é dispensável ao processo de formação do 'cidadão produtivo alienado', ou seja, do “trabalhador que maximize a produtividade sendo um cidadão mínimo"7.

$\mathrm{Na}$ via da formação profissional, sua função de responder às necessidades da sociedade, na qual está inserida, faz instituir em seu contexto os subsídios para que as relações sociais, inclusive com o mundo do trabalho, ocorram em sintonia com cada momento histórico na forma de uma equação cujos componentes envolvem projetos coletivos e individuais. Os coletivos são explicitados pelas políticas governamentais. Os individuais estão representados pelas aptidões e desejos do ser humano que, nem sempre vêm ao encontro das demandas do coletivo. Uma história marcada, também, pela "dualidade estrutural"6, onde a escola primária e profissionalizante estaria destinada aos pobres para 'formar a classe dos trabalhadores manuais', enquanto a escola propedêutica e superior, caminho dos mais abastados da sociedade, 'formaria a classe dos trabalhadores intelectuais'6. Essa dualidade, produto da lógica capitalista, forjou na sociedade um estigma, uma marca que associou por séculos o ensino de técnicas de produção aos escravos, à pobreza e ao assistencialismo.

O que a história nos mostra é um cenário onde a formação de técnicos se associou à valorização enfática do conhecimento específico, direcionado para o sustento do modo de produção capitalista. Cabe ressaltar que esse contexto é ainda mais grave no caso da saúde, cujo trabalho se caracteriza pelas incertezas decorrentes da indeterminação das demandas, pela descontinuidade e pela disponibilidade para atender a todos os casos, inclusive aqueles excepcionais.

Inserida nesse contexto, a formação dos A\&TE não é exceção, pois “a educação profissional em saúde não se caracterizou historicamente por uma síncrese, mas se pautou, hegemonicamente, pelo referencial epistemológico do prag-

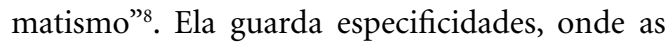
decisões a serem tomadas implicam em desenvolver uma formação que expresse, essencialmente, a "capacidade de um ser humano cuidar de outro" ". Assim, inserida nas contradições históricas existentes nas "relações sociais de explo-

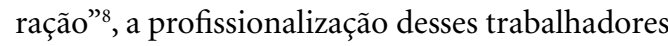
via cursos técnicos de nível médio, que poderia, por vocação, animar e enobrecer, passa a ter o gosto amargo da insuficiência, da incompletude para a sua carreira e sobrevivência.

Portanto, se a formação continuada pode ser considerada complementar e necessária para as atividades laborais, em especial no atendimento às demandas da saúde, o forte investimento dos técnicos em cursos de ensino superior (graduação e pós-graduação) pode representar a influência dos princípios da "teoria do capital humano" nos processos seletivos. Em outras palavras, o domínio do saber profissional está sendo substituído pela mensuração dos cursos realizados, logo, o técnico que dispuser de mais ou maior titulação, como valor agregado a sua formação, é selecionado para a vaga de emprego. Essa realidade expõe as iniquidades das relações estruturais do trabalho que, dadas também na esfera da formação, podem ser traduzidas como a roupagem imposta pelo capitalismo ao mascarar as desigualdades sociais associando escolarização ao emprego. "É sob a égide da "teoria" do capital humano que se traçam planos, diretrizes e estratégias educacionais, especialmente para os países de capitalismo dependente, e se afirma a ideia de que a ascensão e a mobilidade social têm um caminho garantido via escolaridade, mediante empregos bem remunerados" ${ }^{10}($ p.232), mantendo a relação hierárquica de poder estruturalmente predeterminada pelo capital ${ }^{11}$.

\section{Metodologia}

Trata-se de um estudo analítico de variáveis do banco de dados da $\mathrm{PPEB}^{2}$, em que se procura estabelecer relações entre os dados de formação e distribuição dos A\&TE nos estados com o fenômeno da sobrequalificação entre esses profissionais, desvelado na análise e apresentação dos dados da pesquisa referentes ao Brasil.

$\mathrm{A} \mathrm{PPEB}^{2}$ foi realizada pela FIOCRUZ, em pareceria com o Conselho Federal de Enfermagem (COFEN), e já teve seus aspectos metodológicos publicados ${ }^{12}$. As informações utilizadas nesse estudo fazem parte do Banco de Dados, originado pela pesquisa, de acesso público irrestrito, disponível on line na página eletrônica do COFEN.

O estudo buscou relacionar as variáveis 'ano de formação' e 'tipo de entidade mantenedora da instituição/escola em que o curso de auxiliar/técnico foi realizado' para determinar, numa perspectiva histórica, o perfil de formação de A\&TE. Essas informações foram contextualizadas, quando necessário, com dados sobre matrículas, vagas e formandos que constam do Banco de Microda- 
dos dos Censos Escolar (1999-2017) ${ }^{13}$ e da Educação Superior $(2000-2012)^{14}$, disponibilizados no sitio do Inep.

Para comparar os dados da pesquisa sobre a distribuição de A\&TE no território brasileiro, considerando os 26 estados e o Distrito Federal (capital e interior), foram utilizadas informações do IBGE $^{15,16}$, de 2013, ano em que foi iniciada a coleta de dados da PPEB$^{2}$.

Para complementar a análise, estendendo-a até 2017, foram utilizadas as informações da Plataforma Nilo Peçanha (MEC) ${ }^{17}$, ambiente virtual que destina-se à coleta, validação e disseminação das estatísticas oficiais da Rede Federal de Educação Profissional, Científica e Tecnológica. Assim propusemo-nos a avaliar a evolução da oferta de cursos técnicos de enfermagem na Rede Federal, no sentido de identificar se estão sendo criados cursos nos estados em que os dados da PPEB $^{2}$ indicam maior déficit ou se a capacidade educacional instalada está sendo reforçada pelo poder público federal onde já existe concentração significativa de A\&TE, muitas vezes sobrequalificados.

Para mensurar a sobrequalificação em cada estado, foram atribuídos valores para três níveis de escolaridade - estratégia para tratar essa variável como quantitativa. Assim, as variáveis categóricas 'primeiro grau incompleto/completo'; 'segundo grau incompleto/completo'; 'nivel superior incompleto/completo' definidas na $\mathrm{PPEB}^{2}$, foram substituídas por variáveis numéricas.

Nesse sentido, às categorias 'primeiro grau incompleto/completo' e 'segundo grau incompleto' níveis de escolaridade que estão aquém da atual exigência mínima para o registro no Conselho profissional - foi atribuído o valor zero. Às categorias 'segundo grau completo' e 'superior incompleto' - que correspondem à certificação da escolaridade mínima exigida - foi atribuído o valor 100; e o valor 200 à categoria 'superior completo'.

Esse artifício foi para compreender as frequências de escolaridade numa distribuição de tendência central, sendo definida, hipoteticamente, uma condição ideal em que o desvio da curva à esquerda - pequeno, por conta da aposentadoria dos antigos atendentes e auxiliares que não tinham o nível médio e da exigência atual para registro nos Conselhos - é similar ao desvio da curva à direita - também pequeno, porque o A\&TE que atinge novo patamar tende, nessa realidade 'ideal', a abandonar a ocupação de nível médio, substituindo-a por uma nova identidade profissional.

Seguindo esse raciocínio, a medida da assimetria da curva, à direita ou à esquerda, seria capaz de indicar o balanço dos extremos de esco- laridade, ou seja, se no estado em análise há um maior número de auxiliares e técnicos que não possuem o nível médio completo ou com nível superior de escolaridade. Para mensurar esse fenômeno foi empregado o cálculo do Coeficiente de Assimetria de Pearson (CAP), definido por:

$$
\mathrm{CAP}=\frac{3 x(\text { MÉDIA }- \text { MEDIANA })}{\text { desvio padrão }}
$$

Nessas condições, quanto mais alta for a concentração de A\&TE com nível médio, e mais semelhantes forem os contingentes dessas categorias nos extremos (escolaridade inferior ao Ensino Médio e Nível Superior completo ou mais), mais próximo de zero será o CAP calculado para esse estado. Se o CAP for maior do que 1 ou se o CAP for menor do que -1, a distribuição da variável pode ser considerada significativamente assimétrica com cauda à direita ou significativamente assimétrica com cauda à esquerda, respectivamente.

$\mathrm{Na}$ verificação por estado, de forma geral, se o ${ }^{\circ}$ de A\&TE sem o nível médio $>n^{\circ}$ de A\&TE com nível superior completo, quanto maior for a diferença entre esses contingentes, mais afastados de zero e negativos serão os valores do CAP - tratando-se, então, de potencial indicador de subqualificação do contingente de A\&TE no estado em questão. No sentido oposto, se o $n^{\circ}$ de A\&TE sem o nível médio $<\mathrm{n}^{\circ}$ de A\&TE com nível superior completo, quanto maior for a diferença entre esses contingentes, mais afastados de zero e positivos serão os valores do CAP - tratandose, de forma similar, de uma potencial indicação de sobrequalificação do contingente estadual de A\&TE em análise.

No sentido de testar as hipóteses mais comuns que poderiam justificar sobrequalificação num determinado estado, foi testada a correlação das variáveis da $\mathrm{PPEB}^{2}$ rendimentos totais auferidos através de trabalho como A\&TE e entidades mantenedoras das Instituições de Ensino Superior - IES que formaram os enfermeiros de cada estado com o CAP. Assim, empregamos o teste do Coeficiente de Correlação de Spearman (CCS) para verificar a dependência estatística entre as classificações de duas variáveis. Quando a classificação relativa $\left(1^{\circ}\right.$, $2^{\circ}, 3^{\circ}, 4^{\circ}$ etc.) dos valores no interior de duas variáveis for igual, o CCS será igual a +1 . Na medida em que uma ou mais posições relativas for discrepante entre as duas variáveis, o valor do CCS diminuirá e será igual a -1 se as duas variáveis tiverem uma classificação completamente oposta. Assim, quanto mais próximo de zero o valor de CCS, menor a correlação entre as variáveis. 


\section{Resultados}

\section{Entre a Abundância e a Escassez: distribuição de Auxiliares e Técnicos de enfermagem no Brasil}

Os dados do Inep ${ }^{13,14}$ indicam que houve uma concentração de cursos na área da saúde no Sudeste, representando quase a metade do total de matriculados no país. No nível superior, a região soma $48,2 \%$ dos graduados em enfermagem, e na formação de auxiliares e técnicos $46,5 \%$ desses profissionais. No extremo oposto, o Norte apresenta $5,6 \%$ da formação dos enfermeiros e 7,8\% de A\&TE.

De forma geral, e em concordância com os dados de formação obtidos na $\mathrm{PPEB}^{2}$, as informações dão conta de que as políticas públicas não têm sido efetivas no sentido de desconcentrar o aparelho formador de enfermagem, seja em nível superior ou técnico, da região Sudeste e de financiar, com dinheiro público, a formação - em nível técnico, Graduação e Pós-Graduação - dessa Força de Trabalho fundamental para o SUS. O Quadro 1 mostra que a distribuição de A\&TE nos estados não acompanha a distribuição proporcional da área territorial, nem da população entre eles.

Outra característica relevante na distribuição territorial de A\&TE (Quadro 1), é a superconcentração de enfermeiros nas capitais. Verifica-se que, com exceção de 8 estados, a maior parte dos A\&TE está concentrada nas capitais. Vale registrar que Manaus, capital do Amazonas, tem a maior concentração desses profissionais $(89,6 \%)$, contudo, concentra também $52,1 \%$ da população do estado.

Com maior superconcentração de A\&TE destacam-se as capitais dos seguintes estados: Santa Catarina (35\% de A\&TE para 6,8\% da população do estado); Minas Gerais (53,6\% para $12 \%)$; Bahia (75,2\% para $19,2 \%)$; Maranhão (60,3\% para 15,5\%); Rio Grande do Sul (49,2\% para $13,2 \%)$; Pará $(60,8 \%$ para $17,9 \%)$; Paraíba (65,85\% para $19,7 \%)$; Mato Grosso $(58,9 \%$ para $17,9 \%)$ e Curitiba (52,8\% para 16,8\%).

Da mesma forma, ponderando sobre os números de A\&TE em cada estado e respectiva capital, como parâmetro assistencial, ou seja, considerando o número de profissionais por mil habitantes - calculado com base nos dados da $\mathrm{PPEB}^{2}$ e em informações relativas ao contingente populacional ${ }^{16}$ (Quadro 1) - as desigualdades intraestaduais aparecem e podem ser ainda maiores do que as interestaduais ou inter-regionais.
Dessa forma, Roraima, por exemplo, com a menor taxa de A\&TE/1.000 habitantes $(3,87)$, e sua capital (Boa Vista), com uma realidade diferente $(5,19)$. As maiores diferenças entre essas taxas são observadas entre Belo Horizonte $(26,26)$ e Minas Gerais $(5,90)$; Salvador $(20,19)$ e Bahia $(5,15)$; Florianópolis $(19,81)$ e Santa Catarina $(5,82)$; e Belém $(19,69)$ e Pará $(5,79)$.

Por outro lado, importa notar que a participação relativa de auxiliares e técnicos na equipe de enfermagem é, de maneira geral, menor nas capitais do que nos estados, como um todo. Isso significa dizer que, a maioria dos interiores do país possuem uma assistência baseada e dependente desses profissionais, ou seja, de maneira geral uma assistência pior qualificada do que aquela encontrada nas capitais, onde a participação de enfermeiros na equipe é, relativamente, maior. Apenas nas capitais do Amazonas, Bahia, Rio Grande do Norte, São Paulo, Paraná, Santa Cantarina e Mato Grosso do Sul há uma maior participação relativa do A\&TE do que no estado como um todo.

\section{Expansão da Escolaridade: aumento do número de formados e do nível da formação}

No atual contexto de constantes transformações, a área da saúde vem mantendo o diferencial de não substituir o trabalhador pela tecnologia de forma acelerada, apesar de ser um setor de intensa, profunda e acelerada incorporação tecnológica. Esse perfil tende a atrair cada vez mais profissionais que, em busca de formação para atuar no segmento, estimulam o crescimento da oferta de cursos pelo país. A enfermagem, enquanto contingente hegemônico na saúde, é um bom reflexo deste cenário. Entre os anos de 2000 e 2012 foram criados 684 novos cursos de enfermagem, passando de 183 para 867 no período, representando um aumento de $450 \%$ do número de vagas e $750 \%$ do número de concluintes ${ }^{14}$.

Corroborando com esta tendência de busca pela qualificação, segundo dados da $\mathrm{PPEB}^{2}$, cerca de $28,5 \%$ dos A\&TE no país já fizeram ou estão cursando o ensino superior, sendo que destes, $63,7 \%$ na área de enfermagem. Isso significa dizer que do total de A\&TE em atividade no país, $18,1 \%$ cursaram ou estão cursando na área ${ }^{2}$.

Vários aspectos se somam, numa realidade polifacetada, multideterminada e complexa, na determinação do fenômeno que recebeu a alcunha de superqualificação ou sobrequalificação: (1) as incertezas do mercado que geram a sensação de necessidade, no trabalhador, da apro- 
Quadro 1. Auxiliares e Técnicos de Enfermagem no Brasil: população, distribuição e participação na equipe de enfermagem.

\begin{tabular}{|c|c|c|c|c|c|c|}
\hline Estados & Pop. 2013 & $\begin{array}{l}\text { Pop. } 2013^{\mathrm{a}} \text { na } \\
\text { capital (\%) }\end{array}$ & $A \& T^{b}(n)$ & $\begin{array}{l}\text { A\&TE } E^{b} \text { na } \\
\text { capital (\%) }\end{array}$ & $\begin{array}{c}\text { A\&TE na } \\
\left.\text { equipe }^{\text {b }} \%\right)\end{array}$ & $\begin{array}{l}\text { A\&TE na } \\
\text { equipe }^{\text {b }} \text { a } \\
\text { capital (\%) }\end{array}$ \\
\hline \multicolumn{7}{|c|}{ Norte } \\
\hline Acre & 776.463 & 46,0 & 4.604 & 73,7 & 73,3 & 71,3 \\
\hline Amazonas & 3.807 .921 & 52,1 & 32.621 & 89,6 & 79,8 & 80,5 \\
\hline Amapá & 734.996 & 59,5 & 8.778 & 75,6 & 86,8 & 84,9 \\
\hline Pará & 7.969 .654 & 17,9 & 46.163 & 60,8 & 84,1 & 82,0 \\
\hline Rondônia & 1.728 .214 & 28,1 & 10.924 & 38,0 & 79,6 & 79,2 \\
\hline Roraima & 488.072 & 63,3 & 1.887 & 85,0 & 76,8 & 75,7 \\
\hline Tocantins & 1.478 .164 & 17,5 & 10.989 & 41,1 & 74,6 & 74,0 \\
\hline \multicolumn{7}{|c|}{ Nordeste } \\
\hline Alagoas & 3.300 .935 & 30,2 & 14.945 & 61,6 & 78,7 & 75,8 \\
\hline Bahia & 15.044 .137 & 19,2 & 77.412 & 75,2 & 74,2 & 76,3 \\
\hline Ceará & 8.778 .576 & 29,1 & 42.016 & 66,2 & 74,8 & 72,5 \\
\hline Maranhão & 6.794 .301 & 15,5 & 32.221 & 60,3 & 75,3 & 74,5 \\
\hline Paraíba & 3.914 .421 & 19,7 & 22.563 & 65,9 & 71,9 & 70,1 \\
\hline Pernambuco & 9.208 .550 & 17,4 & 56.910 & 52,2 & 77,8 & 73,2 \\
\hline Piauí & 3.184 .166 & 26,3 & 21.301 & 60,3 & 75,9 & 73,7 \\
\hline Rio Grande do Norte & 3.373 .959 & 25,3 & 21.235 & 64,0 & 77,1 & 77,3 \\
\hline Sergipe & 2.195 .662 & 28,0 & 14.184 & 64,1 & 81,6 & 75,7 \\
\hline \multicolumn{7}{|c|}{ Sudeste } \\
\hline Espírito Santo & 3.839 .366 & 9,1 & 33.033 & 21,8 & 78,0 & 72,5 \\
\hline Minas Gerais & 20.593 .356 & 12,0 & 121.544 & 53,6 & 74,7 & 73,9 \\
\hline Rio de Janeiro & 16.369 .179 & 39,3 & 179.769 & 49,9 & 80,9 & 79,8 \\
\hline São Paulo & 43.663 .669 & 27,1 & 348.227 & 63,1 & 77,1 & 77,6 \\
\hline \multicolumn{7}{|c|}{ Sul } \\
\hline Paraná & 10.997 .465 & 16,8 & 60.843 & 52,8 & 76,5 & 80,9 \\
\hline Rio Grande do Sul & 11.164 .043 & 13,2 & 91.591 & 49,2 & 81,8 & 79,4 \\
\hline Santa Catarina & 6.634 .254 & 6,8 & 38.588 & 35,0 & 77,2 & 79,2 \\
\hline \multicolumn{7}{|c|}{ Centro-Oeste } \\
\hline Goiás & 6.434 .048 & 21,7 & 34.943 & 45,7 & 75,4 & 69,1 \\
\hline Mato Grosso do Sul & 2.587 .269 & 32,2 & 13.773 & 53,6 & 75,1 & 75,7 \\
\hline Mato Grosso & 3.182 .113 & 17,9 & 16.227 & 58,9 & 72,2 & 70,4 \\
\hline Distrito Federal & 2.789 .761 & 100,0 & 32.532 & 21,1 & 75,0 & 54,8 \\
\hline
\end{tabular}

A\&TE = Auxiliares e Técnicos de Enfermagem.

Fontes: (a) IBGE; (b) $\mathrm{PPEB}^{2}$. Elaboração própria.

priação de um elenco de variadas e diferenciadas competências profissionais que, materializadas no cotidiano, poderiam representar a diferença entre estar empregado ou não; (2) a necessidade real - de mercado - por um diploma, que vai se tornando requisito para o emprego; e (3) a ampliação da oferta de vagas em IES para setores mais empobrecidos da população, principalmente por faculdades privadas, movidas pelo empresariado da educação. Pode-se somar a isso o fato dos Conselhos Regionais de Enfermagem terem deixado de fornecer o registro profissional a auxiliares, $o$ que pode ser visto pelo trabalhador como uma tendência a exigência de uma escolaridade maior.

Assim, como era esperado, o cálculo do CAP apresentou como resultado o predomínio do coeficiente positivo em 24 estados e DF (Quadro 2), o que denota a existência de um contingente de auxiliares e técnicos sobrequalificados nessas localidades. Apenas no Maranhão e Rio Grande do Sul o CAP ficou ligeiramente negativo, denotando que o contingente sem o nível médio de escolaridade ainda é maior do que aquele dos que já concluíram a graduação. 
Quadro 2. CAP e variáveis da PPEB utilizadas no teste de correlação.

\begin{tabular}{|c|c|c|c|}
\hline Estados & $\mathrm{CAP}^{\mathrm{a}}$ & $\begin{array}{c}\text { IES Públicas na formação } \\
\text { de nível superior em } \\
\text { enfermagem }^{\text {b }}(\%)\end{array}$ & $\begin{array}{l}\text { Média salarial total } \\
\text { auferida por A\&TE., }\end{array}$ \\
\hline \multicolumn{4}{|c|}{ Norte } \\
\hline Acre & 0,8 & 75,0 & 2,8 \\
\hline Amazonas & 0,6 & 42,8 & 2,7 \\
\hline Amapá & 0,5 & 72,3 & 3,3 \\
\hline Pará & 0,5 & 72,4 & 2,9 \\
\hline Rondônia & 0,2 & 24,4 & 2,7 \\
\hline Roraima & 0,6 & 46,7 & 3,0 \\
\hline Tocantins & 1,2 & 21,1 & 2,8 \\
\hline \multicolumn{4}{|c|}{ Nordeste } \\
\hline Alagoas & 0,4 & 54,6 & 2,4 \\
\hline Bahia & 0,2 & 38,9 & 3,2 \\
\hline Ceará & 0,3 & 47,9 & 2,3 \\
\hline Maranhão & $-0,1$ & 40,5 & 2,2 \\
\hline Paraíba & 0,5 & 36,1 & 2,6 \\
\hline Pernambuco & 0,5 & 51,9 & 2,7 \\
\hline Piauí & 0,7 & 61,8 & 2,5 \\
\hline Rio Grande do Norte & 0,7 & 67,0 & 2,8 \\
\hline Sergipe & 0,6 & 58,2 & 2,4 \\
\hline \multicolumn{4}{|c|}{ Sudeste } \\
\hline Espírito Santo & 0,3 & 22,0 & 2,5 \\
\hline Minas Gerais & 0,1 & 25,2 & 2,8 \\
\hline Rio de Janeiro & 0,8 & 40,6 & 3,6 \\
\hline São Paulo & 0,4 & 24,8 & 3,7 \\
\hline \multicolumn{4}{|c|}{ Sul } \\
\hline Paraná & 0,6 & 37,0 & 2,9 \\
\hline Rio Grande do Sul & $-0,04$ & 37,6 & 3,1 \\
\hline Santa Catarina & 0,4 & 36,8 & 3,5 \\
\hline \multicolumn{4}{|c|}{ Centro-Oeste } \\
\hline Goiás & 0,4 & 17,5 & 2,9 \\
\hline Mato Grosso do Sul & 0,5 & 40,8 & 2,9 \\
\hline Mato Grosso & 0,5 & 43,0 & 2,7 \\
\hline Distrito Federal & 1,1 & 38,0 & 4,5 \\
\hline
\end{tabular}

(a) Coeficiente de Assimetria de Pearson; (b) Fonte: $\mathrm{PPEB}^{2}$; (c) Média, em salários mínimos, dos rendimentos totais auferidos em todos os trabalhos como auxiliar ou técnico de enfermagem (salário mínimo/2013 = R \$680,00); A\&TE. = Auxiliares e Técnicos de Enfermagem.

Fonte: Elaboração própria.

No Gráfico 1 a dispersão dos valores do CAP evidencia tendências de sub e sobrequalificação por estado. O que fica evidente, no quadro geral, é a sobrequalificação como a tônica dos mercados na maioria dos estados, mas destaque deve ser dado ao Distrito Federal e Tocantins, únicos com o indicador maior do que 1 (um), ou seja, uma curva de distribuição considerada significativamente assimétrica com cauda à direita, o que representa um contingente de A\&TE com escolaridade acima da exigida para o desempenho de suas atribuições significativamente maior do que o contingente de A\&TE sem a formação mínima atualmente exigida.

Um dos aspectos que, possivelmente, influenciam a tendência a sobrequalificação de um contingente de trabalhadores é o acesso à universidade pública. Nesse sentido utilizamos a participação relativa de IES públicas na formação de enfermeiros - uma das variáveis da $\mathrm{PPEB}^{2}$ como um indicador de acessibilidade e avaliamos sua correlação com o CAP - nesse estudo, tratado como um indicador de sub ou sobrequalificação dos A\&TE em um estado (Quadro 2). 


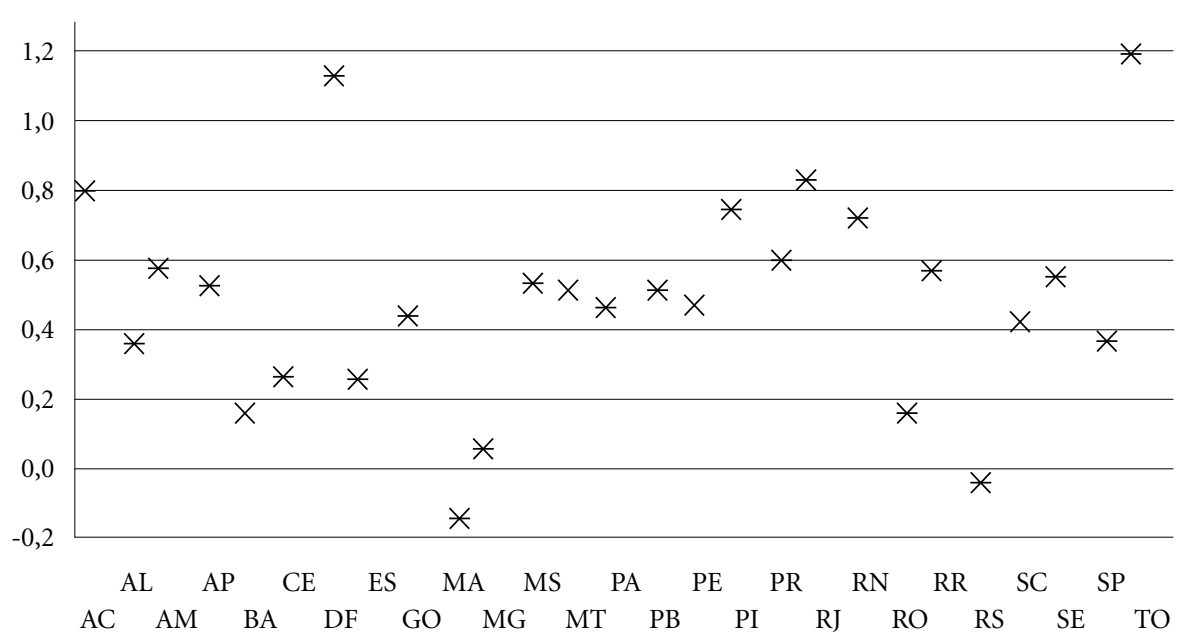

Gráfico 1. Distribuição do indicador de sub e sobrequalificação - CAP por estado - Brasil.

Fonte: PPEB $^{2}$ - 2013, FIOCRUZ/COFEN. Elaboração própria.

Analisando o perfil dos três estados com menores CAP (Quadro 2), o percentual de formação de enfermeiro em IES pública varia de $25,2 \%$ a $40,5 \%$. Já nos três estados com maiores CAP, esse percentual oscila entre $21,1 \%$ a $40,6 \%$. Aplicando o CCS, o valor de rho $=0,312(p$-value $=0,113)$ indica que existe baixa correlação entre vocação estadual para formação de enfermeiro em IES pública e o CAP. Isso pode apontar para um cenário em que uma maior possibilidade de cursar o nível superior em instituição pública não se traduza em fator determinante para a qualificação dos A\&TE.

O mesmo se pode dizer da possibilidade de correlacionar níveis salariais praticados, em média, nos estados para A\&TE, ao indicador de sobrequalificação. Os três com menores CAP ocupam, respectivamente, o último $\left(27^{\circ}\right)$, o $7^{\circ}$ e o $14^{\circ}$ lugares no ranking dos estados que possuem salários médios mais altos para A\&TE. E, para os três com maiores CAP, as posições no referido ranking são $15^{\circ}, 1^{\circ}$ e $3^{\circ}$ lugares, respectivamente. Calculando o CCS, o valor de $r h o=0,195$ $(p$-value $=0,328)$ denota uma baixa e não significativa correlação. Dessa forma, o nível salarial praticado para $\mathrm{A} \& \mathrm{TE}$ parece influenciar, mas não ser determinante da sobrequalificação estadual.

\section{A Privatização na Formação do Auxiliar e do Técnico em Enfermagem e a Participação da Rede Federal}

Nos últimos 40 anos, as instituições privadas de ensino realizaram uma expansão de escolas e vagas em todo o território, assumindo, quase como um monopólio, a formação de profissionais da saúde.

No Censo Escolar de $1999^{13}$, a iniciativa privada era responsável por $62 \%$ da formação técnica de nível médio na área da saúde, enquanto nesse mesmo ano apenas $44 \%$ do total das matrículas da educação profissional de nível técnico em geral eram oriundas do setor privado. A maior participação do setor privado na formação técnica em saúde cresceu, ainda mais, ao longo da década seguinte e, em $2009^{13}$, apenas $24,7 \%$ dos matriculados em cursos técnicos na área da saúde desenvolviam sua formação em escolas técnicas públicas.

Os resultados da $\mathrm{PPEB}^{2}$ reforçam a necessidade de formulação de políticas públicas com potencial de interferir na predominante oferta do setor privado na área da saúde. Os dados da pesquisa $^{2}$ mostram que, ao longo dos anos, as instituições privadas ampliaram sua atuação. Entre os 
anos 1970 e 1979, cerca de 10 mil, ou 61,6\% dos A\&TE, concluíram seus respectivos cursos em escolas privadas (com fins lucrativos: $44 \%$ e sem fins lucrativos: 17,6\%). Entre os anos de 1980 e 1989, o quantitativo de concluintes sobe para cerca de 72 mil, sendo que $62,5 \%$ em escolas privadas (com fins lucrativos: 50,4\% e sem fins lucrativos: $12,1 \%)$. Nas décadas seguintes o quantitativo de egressos cresce e, em 2009, ultrapassa os 600 mil concluintes, tendo as escolas privadas a participação em 83,8\% (com fins lucrativos: 78\% e sem fins lucrativos: 5,8\%). Nos primeiros anos da década seguinte, apesar da redução de concluintes em valores absolutos (pois tratam-se de egressos de apenas 4 anos: 2010 a 2013), o percentual de formados em cursos privados chegou a $88,8 \%$ (Gráfico 2).

Os dados da pesquisa² (Gráfico 2) sugerem que o cenário do perfil de formação não deu sinais de mudanças entre as décadas. A participação das instituições públicas permaneceu sendo bem inferior às do setor privado de ensino. Considerando somente o desempenho da Rede Federal, os dados disponibilizados pelo Censo Escolar $^{13}$ e pela Plataforma Nilo Peçanha ${ }^{17}$ demonstram um certo descolamento das Instituições Federais com as políticas de formação de recursos humanos para a área da saúde. O próprio curso técnico de enfermagem, aparentemente, não é considerado como sendo uma prioridade.

Em 2009, ano posterior a criação da Rede Federal, o Censo Escolar ${ }^{13}$ registrou 2.421 matri- culados nos 26 cursos técnicos de enfermagem oferecidos pelas Instituições Federais. No ano de 2017 esta formação era oferecida em 36 unidades, com um total de 4.294 alunos $^{17}$, ou seja, foram 1.873 novas matrículas, um aumento pouco expressivo se comparado com o crescimento total da Rede Federal entre os anos de 2009 e 2017. Em número absoluto significou um salto de 86 mil para 564 mil matrículas nos cursos de educação profissional ${ }^{13,14,17}$.

Analisando a distribuição das matrículas no curso técnico de enfermagem nos estados, os números indicam um crescimento pouco expressivo no cenário nacional. Apesar do aumento no quantitativo de unidades oferecendo cursos na área da saúde - passando de 51, em 2009, para 113, em $2017^{13,14,17}$ - no período analisado a distribuição dos cursos técnicos de enfermagem se manteve quase que estável. Dentre os 26 estados e o Distrito Federal, que compõem o território brasileiro, em 2009 estes cursos foram oferecidos em 13 estados, com uma concentração em Minas Gerais (6 unidades) ${ }^{13}$. Em 2017, após o período mais intenso da expansão da Rede Federal, o curso técnico de enfermagem estava presente em 16 estados, mantendo Minas Gerais na liderança, com a oferta em 10 unidades. Regionalmente, o Sudeste com 12 unidades e o Nordeste com 11 unidades possuem o maior quantitativo, enquanto o Centro-Oeste, com duas unidades, é a região com o menor índice de oferta do curso técnico de enfermagem pela Rede Federal ${ }^{17}$.

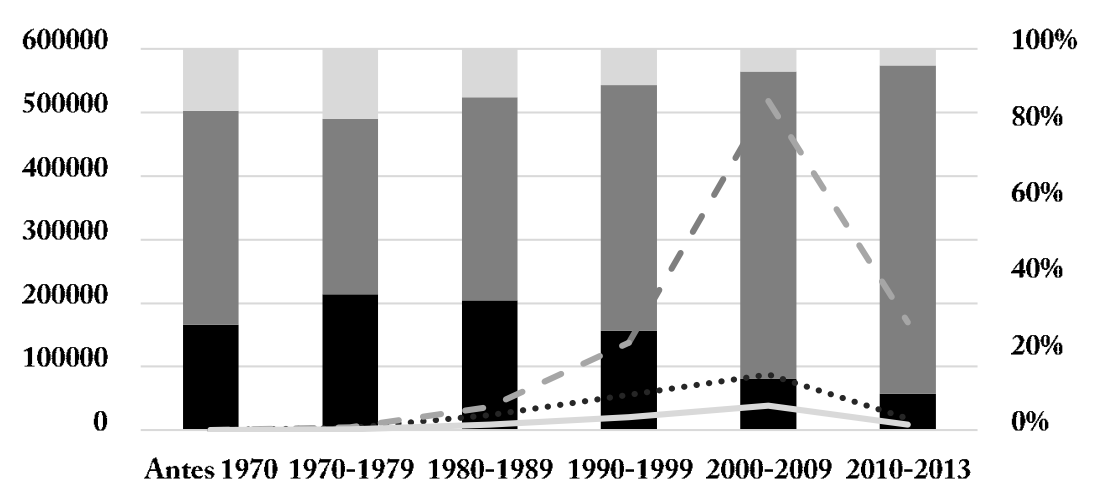

...... Pública - Privada Filantrópica

Gráfico 2. Quantitativo de auxiliares/técnicos de enfermagem por período de formação e tipo de Instituição de Ensino - Pública, Privada e Filantrópica - Brasil. 
Outra questão a ser levantada é a da cobertura. Considerando que um número reduzido de A\&TE/1.000 habitantes é um bom indicador de déficit de formação de auxiliares e técnicos, era de se esperar que houvesse um movimento da Rede Federal no sentido de suprir essas necessidades. Porém, não é isso que se apresenta nos dados; dos 10 estados que possuem um menor número de A\&TE/1.000 habitantes (Quadro 1), apenas Goiás tem dois novos cursos técnicos de enfermagem na Rede Federal entre os anos de 2009 e 2017 $7^{13,14,17}$.

$\mathrm{O}$ que os números têm mostrado, até aqui, representa a ausência das instituições federais na formação do técnico de enfermagem. Considerando que a democratização do acesso aos serviços de saúde requer, necessariamente, a formação dos profissionais que atuarão nestes serviços, a oferta de cursos e a ampliação de vagas pela Rede Federal em todo território nacional são condições sine qua non de cobertura e de acesso universal à saúde. Apesar de não terem sido encontrados estudos ou justificativas sobre os reais motivos que conduzem a Rede Federal a ter pouca oferta de cursos de técnicos de enfermagem, os dados demonstram que o poder público vem negligenciando seu dever de formador de profissionais para o setor da saúde ${ }^{18}$.

\section{Conclusões}

O trabalho e, especialmente, o trabalhador da saúde tem sido encarado como um dos pilares que viabilizam a cobertura e $\mathrm{o}$ acesso universal à saúde, entretanto a realidade ainda está longe da ideal. O fato é que o atual cenário demonstra os "profundos desequilíbrios e lacunas na disponibilidade, distribuição, composição, competência e produtividade dos recursos humanos de saúde, principalmente na atenção primária" ${ }^{19}$. Estas distorções acontecem sob diferentes perspectivas, mas a relação entre infraestrutura necessária ao trabalho e a composição da equipe de saúde exerce uma forte influência na locação dos profissionais, ou seja, a manutenção da capacidade instalada dos serviços de saúde é um dos fatores determinantes para atrair novos profissionais, mantendo a regularidade do acesso à saúde. Outros estudos ${ }^{20,21}$ realizados no Brasil trazem a mesma conclusão, apontando a infraestrutura dos municípios como um fator para a concentração ou dispersão dos profissionais de saúde, incluindo os enfermeiros, técnicos e auxiliares de enfermagem. Os estados da região Sudeste, com destaque para São Paulo, detêm os maiores percentuais de postos de trabalho de todo o país, enquanto os sete estados do Norte, juntos, têm muito menos, cerca de $8,66 \%$ dos postos de técnico de enfermagem e $4,66 \%$ dos postos de auxiliar de enfermagem ${ }^{20}$.

Para agravar este contexto de distorções, a qualificação profissional tem sido um desafio social que perpassa a formação para o trabalho tanto pelo seu nível macro, caracterizado por políticas públicas direcionadas à promoção da formação inicial do profissional, quanto no seu nível micro, marcado pela percepção do indivíduo sobre o momento em que deve aprimorar seus conhecimentos pela via da formação continuada.

Não por coincidência, os estudos ${ }^{20,21}$ já realizados indicam que São Paulo é o estado com maior percentual de postos de trabalho de saúde ocupados e os estados da região Norte como os de menor percentual, na via da formação esse quadro se repete: dos cerca de 1.3 milhões de A\&TE representados na $\mathrm{PPEB}^{2}, 23,3 \%$ se formaram em São Paulo. No extremo oposto, na média a região Norte apresenta os menores percentuais, com destaque para Amapá $(0,1 \%)$, Acre $(0,3 \%)$ e Roraima $(0,5 \%)^{2}$.

Cabe ainda lembrar que dentre as estratégias de ampliação do acesso da população à saúde, a Organização Mundial da Saúde destaca a necessidade do fortalecimento da gestão de "desenvolvimento de recursos humanos em saúde com habilidades que facilitem um enfoque integral da saúde"22. De acordo com o estudo publicado pela Organização Pan-Americana de Saúde (OPAS), Agenda de Saúde Sustentável para as Américas 2018-2030, dos 35 países que compõem as Américas, no final de 2015 pelo menos 17 países tinham planos de ação para recursos humanos em saúde alinhados com as políticas e necessidades de seus sistemas de prestação de serviços de saúde. O estudo identificou que "em média havia 48,7 profissionais de enfermagem por 10.000 habitantes nas Américas em 2015. A maior densidade desses profissionais era encontrada na América do Norte - mais de sete vezes maior que na América Latina e o Caribe (110,9 contra 13,6 por 10.000 habitantes)"'22.

Pelos números analisados podemos afirmar que a realidade dos municípios brasileiros oscila entre perfis semelhantes aos Estados Unidos da América e do Caribe, tanto pela ocupação dos postos de trabalho, quanto na capacidade de formação de profissionais da saúde. Por outro lado, compreender o comportamento dos profissionais frente ao desafio da qualificação permanente e, concomitantemente, o cenário construído pelo 
perfil das oportunidades de formação, é um caminho na direção da construção de políticas que tenham por objetivo a redução das desigualdades regionais na formação de profissionais de enfermagem, um requisito para a ampliação das oportunidades de qualificação dos trabalhadores que lidam com a vida e a saúde da população.

Para finalizar, cabe relembrar o papel de indutora de qualidade no ensino que a Rede Federal deve assumir na saúde - como ocorre em outros setores - quase que naturalmente, por sua presença nacional e relevância no processo de transformação do cenário educacional, caracterizada pela capacidade de disseminação da cultura de excelência e de estruturação de ciclos de melhorias contínuos, configurados a partir de ações de inovação e proposições de políticas.

\section{Colaboradores}

MCMW Wermelinger, A Boanafina, MH Machado, M Vieira, FR Ximenes Neto e WF Lacerda, participaram da concepção e delineamento do estudo, redação e revisão do conteúdo intelectual até a versão final do manuscrito. 


\section{Referências}

1. Fernandes F. Universidade Brasileira: reforma ou revolução? São Paulo: Ed. Alfa-Omega; 1975.

2. Machado $\mathrm{MH}$, coordenadora. Perfil da enfermagem no Brasil: relatório final. Rio de Janeiro: NERHUS DAPS - ENSP/Fiocruz; 2017.

3. Brasil. Conselho Federal de Enfermagem (COFEN). $O$ exercício da Enfermagem nas Instituições de Saúde do Brasil:1982-1983. Rio de Janeiro: COFEN, Associação Brasileira de Enfermagem; 1985.

4. Santos AFT. Pedagogia do mercado: neoliberalismo, trabalho e educação no século XXI. Rio de Janeiro: Ibis Libris; 2012.

5. Ponce A. Educação e luta de classes. São Paulo: Cortez; 2001.

6. Kuenzer AZ. Da dualidade assumida à dualidade negada: o discurso da flexibilização justifica a inclusão excludente. Educ Soc 2007; 28(100):1153-1178.

7. Frigotto G, Ciavatta M. Educar o trabalhador cidadão produtivo ou o ser humano emancipado. Trab Educ Saude 2002; 1(1):45-60.

8. Ramos M. Concepções e práticas pedagógicas nas escolas técnicas do Sistema Único de Saúde: fundamentos e contradições. Trab Educ Saude 2009; 7 (Supl. 1):153-173.

9. Deluiz N. O modelo das competências profissionais no mundo do trabalho e na educação: implicações para o currículo. Boletim Técnico SENAC 2001; 27(3):12-25.

10. Frigotto G. Contexto e sentido ontológico, epistemológico e político da inversão da relação educação e trabalho para trabalho e educação. Rev Contemp Educ $2015 ; 10(20): 228-248$

11. Mészáros I. Para além do capital: rumo a uma teoria da transição. São Paulo: Boitempo; 2011.

12. Machado $\mathrm{MH}$, Oliveira E, Lemos W, Lacerda WF, Aguiar Filho W, Wermelinger M, Vieira M, Santos MR, Souza Júnior PB, Justino E, Barbosa C. Mercado de Trabalho da Enfermagem: aspectos gerais. Enferm Foco 2016; 7(n. esp.):35-53.

13. Instituto Nacional de Estudos e Pesquisas Educacionais Anísio Teixeira (INEP). Microdados Censo Escolar 1999-2017 [página na Internet]. Brasília: INEP; 2019. [acessado 2019 Maio 17]. Disponível em: http://portal.inep.gov.br/web/guest/microdados

14. Instituto Nacional de Estudos e Pesquisas Educacionais Anísio Teixeira (INEP). Microdados Censo do Educação Superior 2000-2012 [página na Internet]. Brasília: INEP; 2019. [acessado 2019 Maio 17]. Disponível em: http://portal.inep.gov.br/web/guest/microdados

15. Instituto Brasileiro de Geografia e Estatística (IBGE). Síntese de indicadores sociais 2013: uma análise das condições de vida da população brasileira. Rio de Janeiro: IBGE; 2016.
16. Instituto Brasileiro de Geografia e Estatística (IBGE). Estimativas da população residente nos municípios brasileiros com data de referência em $1^{\circ}$ de julho de 2013 [Internet]. Rio de Janeiro: IBGE; 2013. [acessado 2019 Fev 12]. Disponível em: ftp://ftp.ibge.gov.br/ Estimativas_de_Populacao/Estimativas_2013/estimativa_2013_dou.pdf

17. Brasil. Plataforma Nilo Peçanha (PNP). Dados estatísticos da Rede Federal de Educação Profissional e Tec nológica (Rede Federal) [Internet]. Brasília: SETEC; 2018. [acessado 2019 Maio 17]. Disponível em: http:// resultados.plataformanilopecanha.org/2018/

18. Machado MH. Trabalho e Emprego em Saúde. In: Giovanella L, Escorel S, Lobato LVC, Noronha JC, Carvalho AI, organizadores. Políticas e Sistema de Saúde no Brasil. 2a ed. Rio de Janeiro: Editora Fiocruz; 2012. p. 259-278.

19. Cassiani SHB. Estratégia para o acesso universal à saúde e cobertura universal de saúde e a contribuição das Redes Internacionais de Enfermagem. Rev Lat Am Enfermagem 2014; 22(6):891-892.

20. Scheffer M, coordenador. Demografia Médica no Brasil v. 2: cenários e indicadores de distribuição. São Paulo: FMUSP, CFM, Cremesp; 2013.

21. Girardi SN, coordenador. Construção do índice de escassez de profissionais de saúde para apoio à Política Nacional de Promoção da Segurança Assistencial em Saúde. Belo Horizonte: NESCON - Faculdade de Medicina/UFMG; 2010

22. Organização Pan-Americana de Saúde (OPAS). Agenda de Saúde Sustentável para as Américas 20182030: Um chamado à ação para a saúde e o bem-estar na região [relatório na Internet]. Washington, DC: OPAS, OMS; 2017. [acessado 2019 Jul 13]. Disponível em: http://iris.paho.org/xmlui/bitstream/handle/ 123456789/49172/CSP296-por.pdf?sequence $=1$ \&is Allowed $=y$

Artigo apresentado em 30/04/2019

Aprovado em 20/08/2019

Versão final apresentada em 20/09/2019 